\title{
Nuclear magnetic resonance measurement of hydrodynamic dispersion in porous media: preasymptotic dynamics, structure and nonequilibrium statistical mechanics
}

\author{
S.L. Codd ${ }^{1,2}$ and J.D. Seymour ${ }^{2,3, a}$ \\ ${ }^{1}$ Department of Mechanical and Industrial Engineering, Montana State University, MT 59717 Bozeman, USA \\ ${ }^{2}$ Center for Biofilm Engineering, Montana State University, MT 59717 Bozeman, USA \\ ${ }^{3}$ Department of Chemical and Biological Engineering, Montana State University, MT 59717 Bozeman, USA
}

Received: 24 May 2012 / Received in final form: 15 August 2012 / Accepted: 30 August 2012

Published online: 23 October 2012 - (C) EDP Sciences 2012

\begin{abstract}
Measurement of displacement time and length scale dependent dynamics by pulsed gradient spin echo nuclear magnetic resonance in porous media directly provides the preasymptotic hydrodynamic dispersion coefficient. This allows for comparison with nonequilibrium statistical mechanics models of hydrodynamics dispersion in porous media. Preasymptotic dispersion data and models provide characterization of porous media structure length scales relevant to transport and are related to the permeability and sample heterogeneity.
\end{abstract}

\section{Introduction}

Nuclear magnetic resonance (NMR) has been an important experimental technique for the characterization of porous media structure and transport processes for over 50 years. This is due to the combination of the noninvasive nature of the technique and the range of chemical, physical and dynamic phenomena for which the NMR signal can be encoded. Recently there have been several excellent reviews on the many facets of NMR analysis of porous media including a topical issue [1], with papers capturing a snapshot of current research applications and detailed reviews on topics ranging from magnetic relaxation time structural characterization [2], to adsorption and advective transport quantitation [3]. A review by Song et al. [4] and proceedings from the magnetic resonance in porous media conferences summarize the current state of the art. Additionally, the fundamentals of the theory and implementation of the NMR methods relevant to measurements in porous media are covered in detail in the new book of Callaghan [5]. Thus, in this article rather than cover material already extensively reviewed, we focus specifically on recent NMR measurement of the displacement time and length scale dependent transport dynamics of hydrodynamic dispersion in porous media using the low order statistical moments of the displacement probability distribution measured by pulsed gradient spin echo (PGSE) NMR. Use of the time-dependent

\footnotetext{
a e-mail: jseymour@coe.montana.edu
}

hydrodynamic dispersion coefficient measured from the mean squared displacement or second moment allows direct comparison to statistical mechanics models of transport.

Theoretical modeling of transport processes in porous media [6] applies physics concepts from probability theory $[7,8]$ and statistical mechanics [9], including percolation theory $[10,11]$ and continuous time random walks [12]. Just as granular materials and colloids have become the "statistical mechanics sandbox" for testing concepts of many body and nonequilibrium statistical mechanics [13] porous media provide a statistical mechanics jungle gym where nonequilibrium statistical mechanics models of the interplay of structure and transport can be explored. Measurement of displacement length and time scale dependent molecular dynamics of hydrodynamic dispersion by NMR can be directly compared to these models. In this overview, data from a number of papers are synthesized to demonstrate a unifying approach based on nonequilibrium statistical mechanics. As much as possible, the nomenclature in the original papers is used, even if this means redefinition of variables referred to earlier in the paper with different nomenclature. This has been done in keeping with our goal of unifying and interpreting several recent papers on NMR of porous media transport whose connection may not be apparent for the broader applied physics porous media readership. The use of the coarse-grained moments of the full displacement probability distribution is demonstrated to provide direct interpretation of hydrodynamic dispersion in terms of system structure. 


\section{Theory of nonequilibrium statistical mechanics of hydrodynamic dispersion in porous media}

Hydrodynamic dispersion refers to the dispersion or spreading of passive scalar tracers in fluid flows due to the interaction of advective velocity fields and molecular diffusion. Modeling of hydrodynamic dispersion is rooted in statistical mechanics, and particularly Brownian motion theory. This approach was initiated with the early work of G.I. Taylor who in 1921 derived what would become known as the Ornstein-Uhlenbeck process [14], for the dispersion of a scalar in a turbulent flow [15], and which we refer to in this paper as the Ornstein-Uhlenbeck-Taylor (OUT) process. The diffusive sampling of a spatial distribution of velocity in laminar capillary flow, hydrodynamic dispersion, was elucidated by G.I. Taylor in 1953 [16]. The connections between hydrodynamic, or Taylor dispersion and stochastic process theory are well established $[17,18]$. Application of the theory of Brownian motion of Einstein, Langevin and Smoluchowski to define dispersivity in porous media was undertaken in 1954 by Scheidegger [7]. A firm fluid mechanical basis connecting capillary and random media approaches was subsequently developed by Saffman $[8,19]$. The result of these developments is modeling of scalar passive tracer transport during flow through a porous medium by the advection diffusion equation (ADE), i.e., Smoluchowski or FokkerPlanck equation, or a corresponding stochastic differential equation of the Langevin type [20]. Modification of these types of models using continuous time random walks (CTRWS) and fractional advection diffusion equations to incorporate non-Gaussian statistics and anomalous diffusion is an area of intense current research $[6,12]$. These models incorporate nonlocal effects due to long ranged space and time correlations in dynamics [21]. Long displacement relative to pore scale, or asymptotic in time dispersion in consolidated granular porous media, is characterized by the scaling of hydrodynamic dispersion coefficient $D_{\perp \text { or } \|}(t \rightarrow \infty) \propto P e^{\alpha}$ with Peclet number $P e=$ $l\left\langle v_{||}\right\rangle / D_{\mathrm{m}}$, the ratio of the rate of advection to the rate of diffusion, in which $l$ is a characteristic system length scale, $\left\langle v_{\|}\right\rangle$is the average axial bulk flow direction velocity and $D_{\mathrm{m}}$ is the molecular diffusion [22]. The scaling with $P e$ of the longitudinal, or axial in direction of mean flow, hydrodynamic dispersion $D_{\|}$at low Reynolds numbers, $R e=l\left\langle v_{\|}\right\rangle / \nu$ with fluid kinematic viscosity $\nu$ depends on the physical mechanisms generating the dispersion. Physical mechanisms include Taylor dispersion due to diffusion across streamlines which scales as $\alpha=2$, pure mechanical mixing of streamlines $\alpha=1$ and hold up dispersion scaling as $P e \ln P e$ which is near $\alpha=1.2$ [22,23]. For many consolidated granular packs a scaling $\alpha \sim 1.4$ at intermediate $P e$ is observed which is an indication of a combination of physical mechanisms of dispersion $[22,23]$. The origin of the scaling behavior of longitudinal dispersion with $P e$ has recently been discussed in the context of CTRWs generated by a power law distribution of displacement lengths [24]. In contrast to the longitudinal dis- persion the transverse hydrodynamic dispersion $D_{\perp}$, perpendicular to the direction of flow in which there is no mean velocity, scales linearly $\alpha=1$, with $P e$ in the majority of data and simulations in the literature [25]. The identification of the appropriate characteristic transport length scale $l$ in a porous media is often difficult due to complicated and hierarchical pore structures.

Preasymptotic hydrodynamic dispersion in porous media is modeled by nonequilibrium statistical mechanics using a projection operator method, as formulated by Cushman et al. [9,26]. The model results in a memory function equation governing the Lagrangian velocity fluctuation $u(t)=v-\langle v\rangle$ autocorrelation function $\Psi(t)=$ $\langle u(t) u(0)\rangle$ :

$$
\frac{\mathrm{d} \Psi(t)}{\mathrm{d} t}=\int_{-\infty}^{t} K\left(t^{\prime}\right) \Psi\left(t-t^{\prime}\right) \mathrm{d} t^{\prime}
$$

Equation (1) provides a general approach in which the form of the memory function $K(t)$ models the system dynamics. The relation between the hydrodynamic dispersion coefficient $D(t)$ and the velocity fluctuation autocorrelation function (VACF) is given by the GreenKubo-Taylor $[14,27]$ relation:

$$
D(t)=\int_{-\infty}^{t} \psi\left(t^{\prime}\right) \mathrm{d} t^{\prime}
$$

where the velocity fluctuation $u_{\|, \perp}(t)$ is in the longitudinal or transverse direction for the respective dispersion coefficient. The classical Ornstein-Uhlenbeck process for Brownian motion with exponential VACF $\Psi(t)=\left\langle u^{2}\right\rangle$ $\exp \left(-t / \tau_{\mathrm{c}}\right)$ is obtained for a Markovian process with a Dirac delta function memory function $K(t)=\delta(t) / \tau_{\mathrm{c}}$, where $\tau_{\mathrm{c}}$ is the correlation time of the process [26]. This results in a time dependent diffusion, or dispersion coefficient given in the context of the Einstein relation between mean squared displacement and time $\left\langle(Z(t)-\langle Z\rangle)^{2}\right\rangle=$ $2 D(t) t$ as

$$
D(t)=\left\langle u^{2}\right\rangle \tau_{\mathrm{c}}\left[1-\frac{\tau_{\mathrm{c}}}{t}\left(1-\exp \left(-t / \tau_{\mathrm{c}}\right)\right)\right] .
$$

In the long time asymptotic limit relative to the correlation time $t \gg \tau_{\mathrm{c}}$ the dispersion coefficient is time independent and given by the intensity of the velocity fluctuation $\left\langle u^{2}\right\rangle$ and the correlation time $D=\left\langle u^{2}\right\rangle \tau_{\mathrm{c}}$, resulting in the normal diffusion process linear time scaling of the mean squared displacement $[14,26]$.

A general approach to the time dependence of $\Psi(t)$ is an infinite continued fraction solution to equation (1) in Laplace space [26]. This results in the exponential VACF and delta function memory function when truncated to first-order and to second-order results in an exponential memory function $K(t)=\omega_{\mathrm{v}}^{2} \exp \left(-t / \tau_{\mathrm{o}}\right)$. A physical interpretation of the exponential memory function is the position evolution of a Brownian harmonic oscillator [20] 
or cage diffusion within a fluctuating cage of surrounding fluid particles in dense fluids [28]. In these contexts $\omega_{\mathrm{v}}^{2}$ represents the force autocorrelation function, given by the second moment of the $\mathrm{VACF}$, and $\tau_{\mathrm{o}}$ the correlation time related to the long time asymptotic relaxation to equilibrium diffusion [26]. Hence for the simple harmonic oscillator, a mass on a spring, $\omega_{\mathrm{v}}^{2}$, is the spring constant over the mass and in the dense fluid model it is given by $\nabla^{2} U(x)$, where $U(x)$ is the intermolecular energy potential. The exponential memory function applied in equation (1) results in a velocity autocorrelation function of the form:

$$
\Psi(t)=\left\langle u^{2}\right\rangle \exp \left(-t / 2 \tau_{\mathrm{o}}\right)\left[\cos (\bar{\omega} t)+\frac{1}{2 \tau_{\mathrm{o}} t} \sin (\bar{\omega} t)\right]
$$

where $\bar{\omega}=\sqrt{\left(\omega_{\mathrm{v}}^{2}-1 / 4 \tau_{\mathrm{o}}^{2}\right)}[26]$. Equation (4) has a negative lobe at a time corresponding to the period of oscillation, due to correlated dynamics induced by the restricting potential.

The minimum of the negative lobe in the VACF corresponds to a maximum in the time dependent diffusion coefficient. In the Brownian harmonic oscillator the minimum in the VACF occurs around the period of the oscillator $1 / \omega=\sqrt{(m / k)}$ while in the fluctuating cage dense fluid model it occurs on the order of the time for a tracer molecule to transit the cage of surrounding fluid molecules, which is determined by the constraining length of the intermolecular potential and the fluid diffusivity $[26,28]$. In flow in porous media $\omega_{\mathrm{v}}^{2}$ is related to the pore structure constraining effect and curvature of the pore scale fluid particle pathlines, coincident with streamlines for short displacements in which negligible hydrodynamic dispersion occurs and stationary Eulerian flow [29], determined by the pore scale velocity field. In a simple toy model of the flow a sinusoidal velocity at the pore scale is superimposed on dispersive flow over many pore lengths, as proposed by Callaghan and Codd [30]. The sinusoidal flow is induced by the pore structure. As an example consider an array of ordered cylinders, or a crystalline sphere pack in $2 \mathrm{D}$, of diameter $d$ which generates a sinusoidal flow with longitudinal flow along the $x$-direction, the transverse component can be approximated with the form $v_{y}=v_{\mathrm{o}} \cos (y / L)$, where $L$ is the period of the flow and $v_{\mathrm{O}}$ is a characteristic velocity. Then the time scale when the velocity is correlated with itself is $\tau_{\mathrm{v}} \sim\left(L / v_{\mathrm{o}}\right)$, where the period of the flow is $L=2 d$. The velocity is anticorrelated with itself at time corresponding to the negative lobe of the velocity autocorrelation function at time $\tau_{\mathrm{v}} \sim\left(d / v_{\mathrm{o}}\right)$ and the minimum in the velocity autocorrelation provides the length scale $d$ of the structure which induced the oscillatory flow [31]. Thus, the exponential memory function $K(t)=\omega_{\mathrm{v}}^{2} \exp \left(-t / \tau_{\mathrm{o}}\right)$ provides a simple coarse-grained model for the preasymptotic transverse hydrodynamic dispersion which directly connects a maximum in $D_{\perp}(t)$ to a pore scale structure induced transport length scale [32].

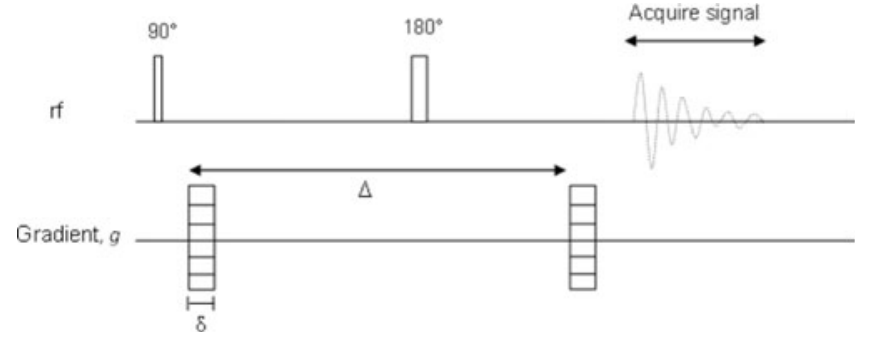

Fig. 1. The PGSE NMR pulse sequence indicating the time sequence for radio frequency $(r f)$ and magnetic field gradient pulses to encode for displacement over experimental time $\Delta$. The $90^{\circ}$ rf pulse excites the first magnetization which is then encoded for position of the NMR active nuclei by the magnetic field gradient pulse of amplitude $g$ and duration $\delta$ during the phase evolution period before the $180^{\circ} \mathrm{rf}$ pulse. After the $180^{\circ} \mathrm{rf}$ pulse, the sense of phase evolution of the magnetization is reversed and the second gradient pulse unwraps the phase encoded by the first gradient. This encodes the NMR voltage signal for displacement as mathematically described in the text.

\section{Experimental pulsed gradient spin echo NMR}

The measurement of diffusion using pulsed magnetic field gradients provides a means to measure displacement dynamics of NMR active nuclei over experimentally controlled displacement wavelength and observation time scales. The simplest form of the experiment is shown in the pulse sequence of Figure 1 which shows the time sequence of the application of radio frequency $(r f)$ pulses and pulsed magnetic gradients applied to measure displacement dynamics [5]. More complex combinations of $r f$ and gradient pulses can be used to encode multidimensional displacement correlations [33] and dynamics including the full nonlocal dispersion correlations $[5,34]$. In porous media with significant magnetic susceptibility between the solid and fluid phase, such as some geologic media, more advanced pulse sequences using combinations of $r f$ and gradient pulses to refocus susceptibility-induced dephasing of the NMR signal are required [35]. The measured voltage signal in the PGSE NMR experiment normalized to eliminate the effects of magnetic relaxation is

$$
\begin{aligned}
E(q, \Delta) & =\frac{S(q, \Delta)}{S(q=0, \Delta)}=\int P(Z, \Delta) \exp [\mathrm{i} 2 \pi q Z] \mathrm{d} Z \\
& =\langle\exp [\mathrm{i} 2 \pi q Z]\rangle
\end{aligned}
$$

The signal depends on the pulsed gradient amplitude $g$ applied in the $z$-direction and duration $\delta$ through the reciprocal Fourier variable $q=(2 \pi)^{-1} \gamma g \delta$ to displacement $Z(\Delta)=z(\Delta)-z(0)$ over displacement time $\Delta$ determined by the time separation of the magnetic field gradient pair [5]. Here, $\gamma$ is the gyromagnetic ratio. The spin echo signal $E(q, \Delta)$ is the Fourier transform of the average propagator of the motion $P(Z, \Delta)=$ $\int \rho\left(z^{\prime}\right) P\left(Z, \Delta \mid z^{\prime}, 0\right) \mathrm{d} z^{\prime}$ which gives the probability for a displacement $Z$ in time $\Delta$ and is the average over the 
initial distribution of the nuclei $\rho\left(z^{\prime}\right)$ of the conditional probability for the nuclei to be at $z$ at time $\Delta$, given it was at $z^{\prime}$ initially [5]. The applied gradient direction can be changed to encode longitudinal or transverse displacements. The full propagator is a powerful means for characterizing preasymptotic dispersion in porous media [23,33,36,37]. Structural features in ordered porous media manifest themselves as coherence, or diffractionlike features at $l \sim q^{-1}$, in the reciprocal $q$-space and a corresponding displacement length scale features in the propagator $[5,38,39]$. The amount of detailed information in the propagator even in model sphere bead packs often necessitates comparison with numerical simulation to extract meaningful physical insight into the preasymptotic transport process $[37,40]$. In the case of full propagator longitudinal and transverse displacement correlation measurements which exhibit highly complicated structure, Stapf et al. quote a statistical viewpoint that "... the mathematical representation of the relationship, once demonstrated, is an art in itself" $[5,33,41]$. The same is true for interpretation of the full propagator itself in terms of pore structure, connectivity or permeability and other properties and testing of analytical models.

The low order coarse-grained moments of the propagator can provide characterization of the hydrodynamic dispersion transport dynamics $[5,38,42]$. The relationship in equation (6) indicates that the measured PGSE NMR signal is the characteristic function of the probability distribution of the displacement dynamics [14]. The time dependent dispersion dynamics can be obtained by focusing on the low- $q$ regime and applying the cumulant expansion theorem to analyze the measured signal $[5,43]$. The cumulant expansion leads to

$$
\begin{aligned}
E(q, \Delta)= & \langle\exp [\mathrm{i} 2 \pi q Z]\rangle=\exp [\mathrm{i} 2 \pi q\langle Z\rangle] \\
& \times \exp \left[-4 \pi^{2} q^{2}\langle Z(\Delta) Z(0)\rangle_{\mathrm{c}}+\cdots\right]
\end{aligned}
$$

This expansion exhibits oscillations due to the mean velocity and odd cumulants of higher order such as the thirdorder skew. Whereas the signal attenuates due to the second-order cumulant $\langle Z(t) Z(0)\rangle_{\mathrm{c}}=\left\langle(Z(t)-\langle Z\rangle)^{2}\right\rangle$ or mean squared displacement and higher-order even cumulants $[5,42,43]$. A linear fit to the attenuation of $\ln [E(q, \Delta)]$ in the low- $q$ limit as a function of $q^{2}$ provides the displacement time dependent dispersion coefficient $D(\Delta)=\left\langle(Z(\Delta)-\langle Z\rangle)^{2}\right\rangle / 2 \Delta[5]$. The utility of this approach to characterize structure and transport in porous media is demonstrated in the representative work discussed below.

\section{NMR measurement of displacement scale dependent hydrodynamic dispersion}

\subsection{Longitudinal dispersion and packed bed scale heterogeneity}

The displacement time dependent longitudinal dispersion in the direction of flow measured in model packed beds of (a)

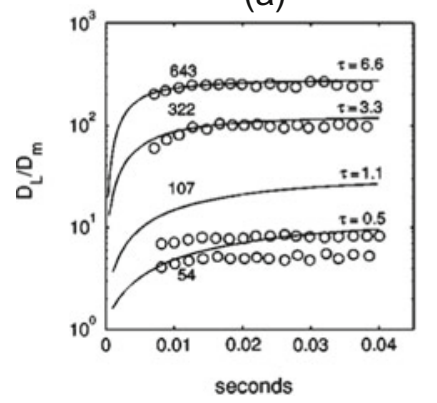

(b)

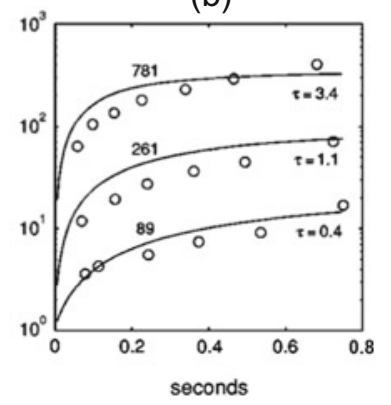

Fig. 2. Longitudinal dispersion $D_{\|}=D_{L}$ normalized by molecular diffusion $D_{m}$ as a function of displacement time $\Delta$ in seconds. (a) A comparison of NMR data from Seymour and Callaghan [23] with simulations using porosity $\varepsilon=0.44$, sphere diameter $d=91 \mu \mathrm{m}, D_{m}=2.13 \times 10^{-9} \mathrm{~m}^{2} / \mathrm{s}$. (b) A comparison of NMR data from Stapf et al. [33] with simulations using $\varepsilon=0.44, d=600 \mu \mathrm{m}, D_{m}=2.1 \times 10^{-9} \mathrm{~m}^{2} / \mathrm{s}$. Values of $P e$ are noted on the plots. Values of $\tau$ denote the elapsed dimensionless time of experiment and simulation. The data demonstrate the linear short-time scaling which asymptotes to a constant value of the classic Brownian motion and Ornstein-Uhlenbeck process. From reference [45].

spheres by NMR shows the classic OUT process behavior as demonstrated in Figure $2[23,33,44,45]$. The NMR data points measured by PGSE NMR are those of Seymour and Callaghan [23] for polystyrene monodisperse $d=91 \mu \mathrm{m}$ spheres at porosity $\varepsilon=0.4450<P e<700$ in Figure 2a and Stapf et al. [33] for glass spheres of $d=600 \mu \mathrm{m}$ at $\varepsilon \sim 0.45$ and $70<P e<800$ in Figure 2b. The data of Seymour and Callaghan show a scaling of longitudinal dispersion with $D_{\perp} \propto P e^{1.4}[23]$. The solid lines in Figure 2 are generated by lattice Boltzmann (LB) simulations [45]. Data and simulation exhibit the linear increase with time of the longitudinal dispersion coefficient $D_{\|}=\left\langle u_{\|}^{2}\right\rangle \Delta$ in the ballistic regime $\left\langle(Z(\Delta)-\langle Z\rangle)^{2}\right\rangle=\left\langle u_{\|}^{2}\right\rangle \Delta^{2}$, at displacement times below the correlation time $\Delta<\tau_{\mathrm{c}}$, and an asymptotic approach to a constant value $D_{\|}=\left\langle u_{\| \mid}^{2}\right\rangle \tau_{\mathrm{c}}$ at times long relative to the correlation time $\Delta \gg \tau_{\mathrm{c}}$. The preasymptotic longitudinal dispersion through the OUT process of equation (3) provides a measure of the Lagrangian velocity fluctuation intensity $\left\langle u_{\|}^{2}\right\rangle$ and the correlation time of the displacement dynamics $\tau_{\mathrm{c}}$.

Preasymptotic longitudinal dispersion allows analysis of the impact of the packing heterogeneity on the hydrodynamic dispersion behavior $[46,47]$. Packing heterogeneity at the macroscale induced, for instance, by wall regions is important for granular packed beds in conduits, commonly found in technological applications such as chromatography and packed bed reactors $[47,48]$. The PGSE NMR approach is based on measurement of the intrinsic dispersivity $l_{\mathrm{d}}=D_{\|} /\left\langle v_{\|}\right\rangle$due to the longitudinal dispersion induced by an exact random packing structure of identical spheres with no macroscale heterogeneity [46]. Heterogeneity in the actual packing is assumed to induce a stationary distribution of velocity variation $P_{\mathrm{v}}(\beta)$, where $\beta=$ $\delta v_{\|} /\left\langle v_{\|}\right\rangle$is the amplitude of the variation in velocity $\delta v_{\|}$within each pore from, and relative to, the 
mean $\left\langle v_{\| \mid}\right\rangle$. This fluctuation in velocity is due not to the "intrinsic" hydrodynamic dispersion as given by $u_{\|}$above, but rather due to the macroscale heterogeneity of the system due to packing variations. In this way the mean squared displacement $\sigma^{2} / 2 \zeta_{\mathrm{o}}=\left\langle(Z(t)-\langle Z\rangle)^{2}\right\rangle / 2 \zeta_{\mathrm{o}}=$ $l_{\mathrm{d}}+\left\langle\beta^{2}>\zeta_{\mathrm{o}} / 2\right.$ normalized by the mean longitudinal displacement $\zeta_{\mathrm{o}}=\left\langle\zeta_{\|}\right\rangle=\left\langle v_{\|}\right\rangle \Delta$ is decomposed into an intrinsic dispersivity $l_{\mathrm{d}}$ and a dispersion contribution from the sample packing heterogeneity which induces a spreading due to the macroscale velocity variance [46].

The experimental separation of the two effects is demonstrated using NMR in Figure 3 [46]. The skew of the displacement dynamics distribution, that is the third order cumulant $\gamma^{3}$, is measured from the imaginary phase oscillated part of the measured PGSE NMR signal [42]. Figure 3 a plots the measured skew normalized by the cube of the root mean squared displacement $\gamma^{3} / \sigma^{3}$ as a function of the mean longitudinal displacement normalized by the sphere diameter $\zeta_{\mathrm{o}} / d[46]$. The data are for three separate packed beds of spheres in $37.5 \mathrm{~mm}$ diameter columns. Packed beds P1 and P3 are composed of $d=100 \mu \mathrm{m}$ beads with porosity $\varepsilon=0.352$ and 0.374 , respectively, and $\mathrm{P} 4$ is composed of $d=500 \mu \mathrm{m}$ beads with porosity $\varepsilon=0.341$ and flow rates corresponding to the $P e$ numbers shown. The authors identify three flow regimes. A velocity distribution smeared by diffusion regime (I) at displacements less than a bead diameter, a transition regime, (II) from displacements just below a bead diameter to near 10 bead diameters and a quasi-asymptotic regime, and (III) beyond 10 bead diameters [46]. The overlap of the skew for P3 and P4 is attributed to mechanical dispersion at the higher $\mathrm{Pe}$ of these data, which is absent at the lower $P e$ of packed bed P1 [46]. Using the skew of the displacement probability distribution to identify the different flow regimes allows the mean squared displacement data plotted versus the sphere diameter normalized mean displacement Figure $3 \mathrm{~b}$ to be used to determine the intrinsic dispersivity $l_{\mathrm{d}}$ from the intercept of a line fit through the quasi-asymptotic regime (III) data. The results indicate that the correct scaling for the system length scale is the hydrodynamic length $\lambda=l=d \varepsilon /(1-\varepsilon)$ and excellent agreement is shown with the random capillary network model of Saffman $[19,46]$. This work uses the skew to identify the approach to asymptotic behavior for which $\Delta \gg \tau_{\mathrm{c}}$. The OUT process behavior analogous to Figure 2 is evident in Figure $3 \mathrm{~b}$ with the mean squared displacement scaling with time squared $\zeta_{\mathrm{o}}^{2}=\left\langle\zeta_{\|}\right\rangle^{2}=\left\langle v_{\|}\right\rangle \Delta^{2}$ in the ballistic regime at times $\Delta<\tau_{\mathrm{c}}$ or $\zeta_{\mathrm{o}} / d<10$ and linearly with time $\zeta_{\mathrm{o}}=\left\langle\zeta_{\|}\right\rangle=\left\langle v_{\|}\right\rangle \Delta$ at long times $\Delta \gg \tau_{\mathrm{c}}$ or $\zeta_{\mathrm{o}} / d>10$.

\subsection{Transverse dispersion and porous media structure}

The transverse dispersion in the direction perpendicular to the bulk flow shows a more complex behavior with a maximum at a certain time and decay to an asymptotic constant $[30,45]$. Callaghan and Codd used a frequencymodulated PGSE method which measures the pulsed gradient frequency dependent dispersion coefficient $D_{\perp}(\omega)=$ (a)

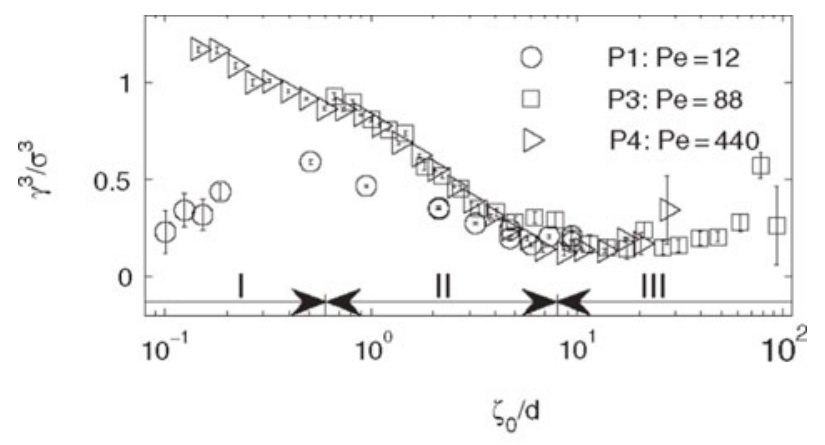

(b)

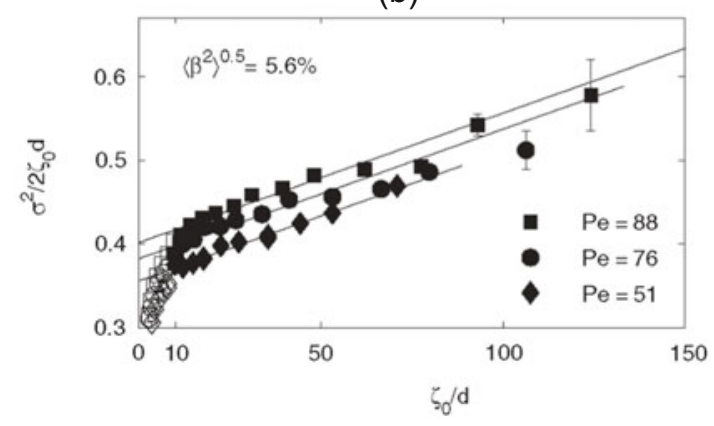

Fig. 3. (a) Skewness factor $\gamma^{3} / \sigma^{3}$, given by the variance, or mean squared displacement $\sigma^{2}$ and skew $\gamma^{3}$ of the displacement distribution, as a function of mean displacement normalized by bead diameter $\zeta_{\mathrm{o}} / d=\left\langle\zeta_{\|}\right\rangle / d$. Three regimes of flow are indicated by the preasymptotic decrease of the skewness factor with displacement length: (I) velocity distribution smeared by diffusion; (II) transition regime; (III) quasi-asymptotic regime. (b) Linear behavior of the normalized mean squared displacement $\sigma^{2} / 2 \zeta_{\mathrm{o}} d$ as a function of the normalized mean displacement $\zeta_{\mathrm{o}} / d$. The solid symbols in the quasi-asymptotic mean displacement regime (III) fitted to $\sigma^{2} / 2 \zeta_{\mathrm{o}}=l_{\mathrm{d}}+\left\langle\beta^{2}\right\rangle \zeta_{\mathrm{o}} / 2$ provide the intrinsic dispersivity $l_{\mathrm{d}}$ as the intercept of the line. Reprinted figure with permission from U.M. Scheven, R. Harris, M.L. Johns, Phys. Rev. Lett. 99 (5) (2007). Copyright 2007 by the American Physical Society.

$\int \psi\left(t^{\prime}\right) \exp \left(i \omega t^{\prime}\right) \mathrm{d} t^{\prime}$, the frequency spectrum of the VACF [49]. The method applies a train of small amplitude $g$ gradient pulses with periodicity $T=1 / \omega$ encoding the NMR signal for displacements as a function of $T$ corresponding to $\Delta$ in the simple PGSE experiment introduced above $[30,49]$. The displacement frequency dependent transverse dispersion measured for packed beds in $2 \mathrm{~mm}$ circular columns of monodisperse spheres of $d=$ 136, 90 and $50 \mu \mathrm{m}$ and porosity $\varepsilon \sim 0.4$ are shown in Figure 4 [30]. The flow regime studied was for Peclet number range of $10<P e<300$ and linear scaling the $D_{\perp} \propto$ $P e$ of the transverse dispersion with $P e$ was observed at low frequency corresponding to long displacement time [ref]. The NMR measured transverse dispersion coefficient exhibits a maximum at intermediate frequency and begins to asymptote to a constant at longer times, i.e., lower $\omega$. The solid lines show an excellent agreement of the NMR data with the simulations of Maier et al. [45]. The simulations exhibit negative lobes in the VACF corresponding to the exhibited maxima in the $D_{\perp}(\omega)$ curves $[30,45]$ 
(a)

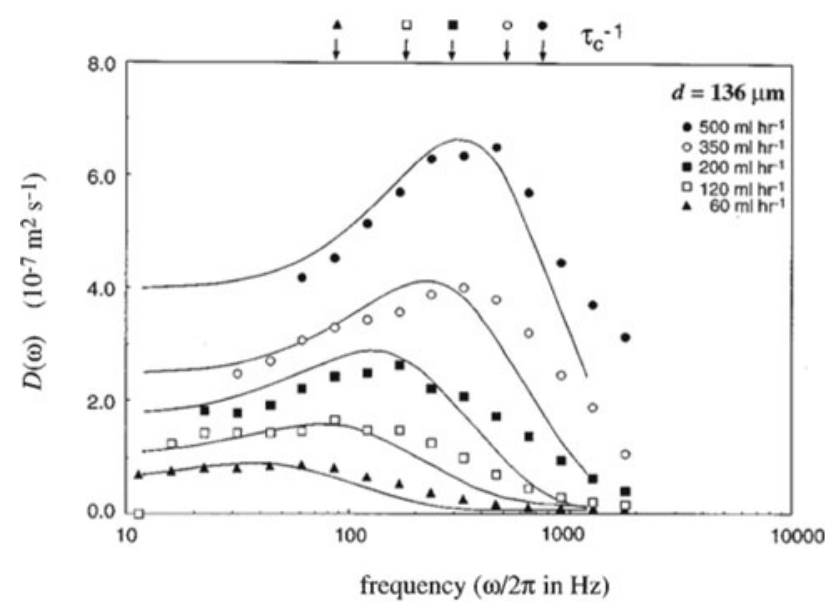

(b)

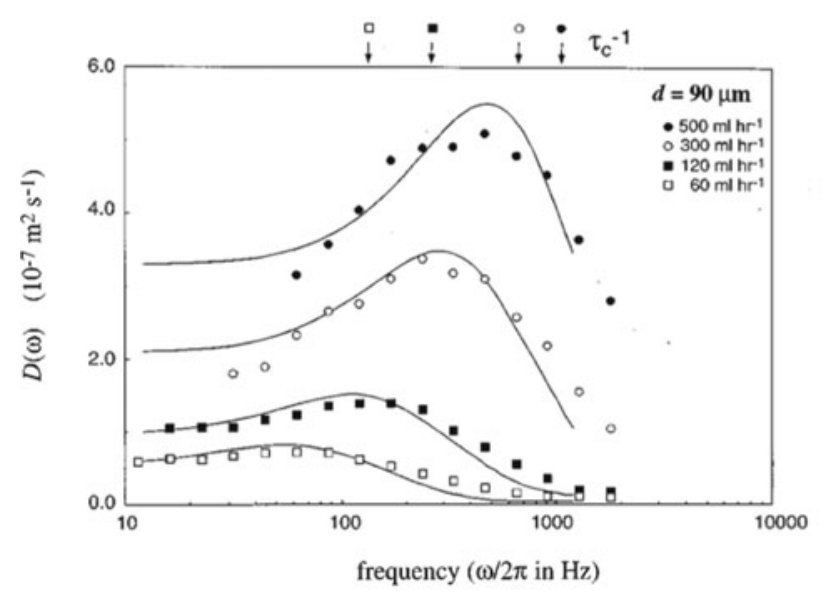

(c)

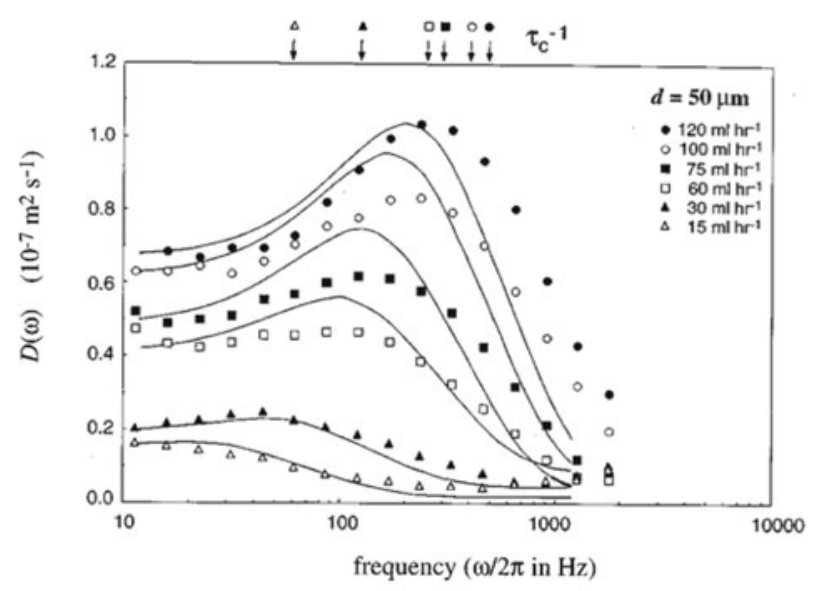

Fig. 4. Effective transverse dispersion coefficients measured by a frequency modulated PGSE NMR method, $D(\omega)$ symbols measured as a function of magnetic field gradient application frequency. Results are for a range of volumetric flow rates as shown for (a) $136 \mu \mathrm{m}$, (b) $90 \mu \mathrm{m}$ and (c) $50 \mu \mathrm{m}$ bead packs. The theoretical fits given by the solid lines are obtained by Fourier transforming the velocity autocorrelation functions of Maier et al. [45]. From Callaghan and Codd [30]. consistent with the analytic exponential memory function model for the VACF. Callaghan and Codd interpreted the data in terms of a simple toy model in which an oscillatory flow due to motion around individual spheres is superimposed on the dispersive mixing flow over multiple pore lengths [30]. The correlation time was taken to be the time $\tau_{\mathrm{v}}=d /\left\langle v_{\|}\right\rangle$to transit a particle diameter $d$ due to the mean velocity $\left\langle v_{\|}\right\rangle$which gives the frequency of the oscillatory flow as $2 / \tau_{\mathrm{v}}$. This simple model is analogous to the oscillatory Brownian harmonic oscillator or exponential memory function model for the VACF, as discussed in Section 2.

The correlation between structure and preasymptotic transverse dispersion dynamics evidenced by the model sphere packs of Callaghan and Codd [30] is not limited to granular packing porous media. PGSE NMR measurement of transverse dispersion in an open cell polymer foam for which porosity is much larger $\varepsilon>0.9$ exhibits the same behavior as is demonstrated in the data of Figure 5 [32]. The transverse dispersion coefficient obtained at varying displacement observation times $\Delta$, normalized by the molecular diffusion of water at four flow rates corresponding to $300<P e<2500$ in a single sample of polyurethane 110 ppi (pores per inch, an industry standard) open cell random polymer foam sample of volume fraction $\varepsilon=0.97$ is plotted as a function of the average longitudinal displacement length $\left\langle\zeta_{\|}\right\rangle=\left\langle v_{\|}\right\rangle \Delta$ in Figure 5a. The scaling of the transverse dispersion $D_{\perp} \propto P e$ is again linear in Peclet number despite the significant structural and porosity difference with granular packed beds [50]. The solid lines are the second-order exponential memory function model with parameters ranging from $\omega_{\mathrm{v}}^{2}=31807 \mathrm{~s}^{-2}$ and $\tau_{\mathrm{o}}=0.0117 \mathrm{~s}$ at the highest flow rate of $\left\langle v_{\|}\right\rangle=15 \mathrm{~mm} / \mathrm{s}$ to $\omega_{\mathrm{v}}^{2}=2309 \mathrm{~s}^{-2}$ and $\tau_{\mathrm{o}}=0.0362 \mathrm{~s}$ at the lowest flow rate of $\left\langle v_{\|}\right\rangle=3.7 \mathrm{~mm} / \mathrm{s}$ [50] and the agreement is excellent. In Figure 5b the molecular diffusion normalized transverse dispersion coefficient for two foam samples, the one mentioned above and the same material with $80 \mathrm{ppi}$ and $\varepsilon=0.93$, measured by NMR and an LB simulation of a larger 50 ppi digitized open cell foam [51] are shown. Each exhibits a maximum in $D_{\perp}$ at a different dispersion length.

Figure 6 a shows the transverse dispersion coefficients in Figure 5b normalized by its own maxima as a function of the displacement length scale normalized by a characteristic length scale for each system. The characteristic length scale is determined from the maximum of the $D_{\perp}$ curve such that $\left.l \equiv\left\langle\zeta_{\|}\right\rangle\right|_{(\mathrm{d} D \perp / \mathrm{d}\langle\zeta||\rangle)=0}[32]$. The NMR data for the two different foam samples, the numerical foam, a digital bead pack of spheres of porosity $\varepsilon=0.44$ with dispersion simulated by the LB method and the exponential memory function model, all collapse on to a common curve indicating a universal preasymptotic transverse dispersion behavior related to a characteristic transport length scale $[32,52]$. This provides a means to characterize complex porous media such as foams, where a typical grain size or sphere diameter is not obvious due to varying strut lengths and diameters resulting in cell polygonal shape and size differences, by a single transport length scale 
(a)

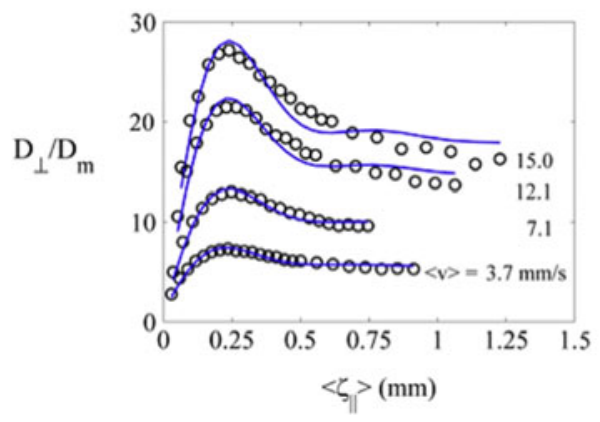

(b)

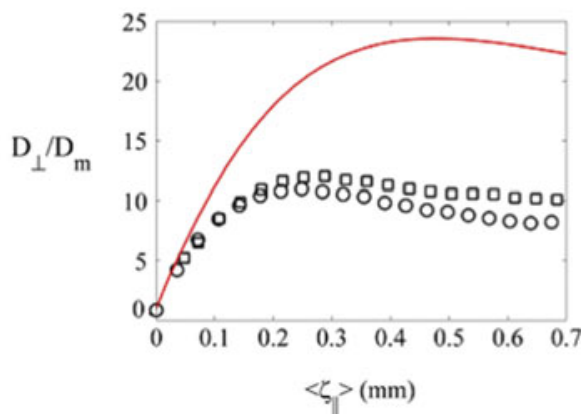

Fig. 5. (Color online) (a) Transverse dispersion coefficient $D_{\perp}$ measured by PGSE NMR (open circles) normalized by molecular diffusion $D_{\mathrm{m}}$ for flow of water through a 110 ppi open cell polymer foam at four different average velocities plotted as a function of mean displacement $\left\langle\zeta_{\|}\right\rangle=\langle v\rangle \Delta$. The NMR data show agreement with fits to the exponential memory function model (blue/solid lines) indicating the correlated dynamics at a displacement where the maxima in $D_{\perp}$ occur. (b) Transverse dispersion coefficient $D_{\perp}$ for LB simulation (red line) of a digital foam structure, NMR data for a 110 ppi foam sample 1 (open circles) at $\left\langle v_{\|}\right\rangle=12.1 \mathrm{~mm} / \mathrm{s}$ and $80 \mathrm{ppi}$ foam sample 2 (open squares) at $\left\langle v_{\|}\right\rangle=8.3 \mathrm{~mm} / \mathrm{s}$ indicating the change in the maxima location with foam structure.

(a)

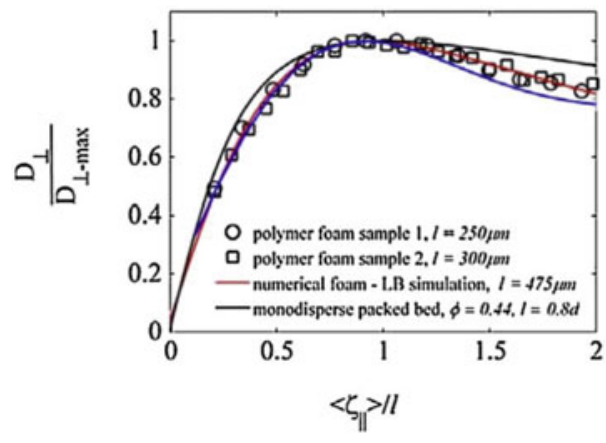

(b)

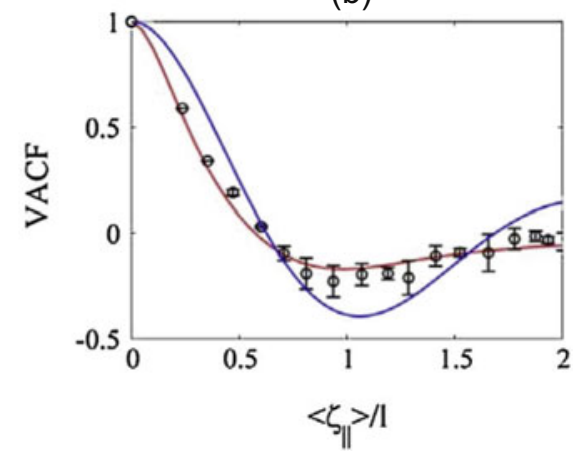

Fig. 6. (Color online) (a) Transverse dispersion coefficient normalized by its maximum amplitude as a function of the mean longitudinal displacement length scaled by the transport length scale $\left\langle\zeta_{\|}\right\rangle / l$ for LB simulation (red line) of a digital foam structure, NMR data for a 110 ppi foam sample 1 (open circles) at $\left\langle v_{\|}\right\rangle=12.1 \mathrm{~mm} / \mathrm{s}$, an 80 ppi sample 2 (open squares) at $\left\langle v_{\| \mid}\right\rangle=8.3 \mathrm{~mm} / \mathrm{s}$, the scaled exponential memory function model (blue line) and LB simulation for a consolidated random sphere packing (black line). (b) The velocity autocorrelation for the average of all the NMR experiments shown in Figure 4a on the 110 ppi foam sample 1 and the nonequilibrium statistical mechanics exponential memory function model (blue line) and L-B simulation (red line). Reprinted figure with permission from T.R. Brosten, S.L. Codd, R.S. Maier, J.D. Seymour, Phys. Rev. Lett. 103, 218001 (2009). Copyright 2009 by the American Physical Society.

related to the hydrodynamic dispersion dynamics [32]. The length scale determined for the packed bed of spheres used in the LB simulation is given by $l=\mathrm{d} \varepsilon /(1-\varepsilon)$ as expected from classic models of transport in packed beds of spheres. It is clear from Figure $6 \mathrm{~b}$ that the maxima in the time dependent transverse dispersion coefficient are due to correlation of the velocity over the characteristic transport length, as indicated by the negative lobe of the transverse VACF. Further advances can be made by development of a rigorous direct connection between the pore scale Eulerian velocity field which has streamlines which oscillate around the solid pore structure and the force correlation $\omega_{\mathrm{v}}^{2}$ of the exponential memory function. This has the potential to allow computational fluid dynamics design of porous media structures to generate controlled transport dynamics and mixing.

\subsection{Permeability and preasymptotic dispersion}

The permeability $k$ of porous media has been a primary means of characterization since the work of Darcy demonstrated the relationship between the mean flow velocity of a liquid of viscosity $\mu$ and the pressure drop $\left\langle v_{\|}\right\rangle=$ $(k / \mu \varepsilon) \nabla p$. The connection between permeability and preasymptotic dispersion has recently been demonstrated by Brosten et al. for dispersion arising from low Re flow in macroscopically homogeneous porous media, using LB simulation and PGSE NMR measurement [53]. The model experimental system was a packed bed of $d=240 \mu \mathrm{m}$ monodisperse polystyrene spheres of porosity $\varepsilon=0.44$ in a $10 \mathrm{~mm}$ cylindrical column [53]. A key component of this work is the general short-time expansion of the dispersion coefficient in terms of a Taylor series expansion of the velocity field. The result is analogous to the 


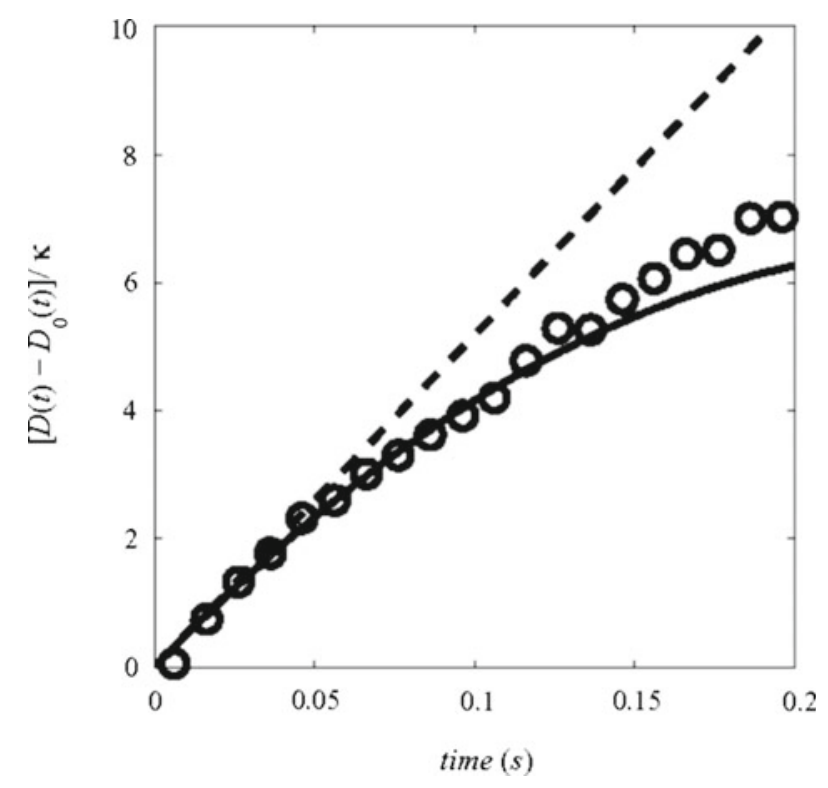

Fig. 7. The difference in time dependent dispersion and diffusion coefficients measured by PGSE NMR (open circles) compared with the linear (dashed line) and quadratic (solid line) short-time predictions for $P e=50.1$ in a consolidated random sphere pack. Parameters of the experiment were $\langle v\rangle=$ $5.7 \times 10^{-4} \mathrm{~m} / \mathrm{s}$, porosity $\varepsilon=0.42$, molecular diffusion $\kappa=$ $D_{\mathrm{m}}=2 \times 10^{-9} \mathrm{~m}^{2} / \mathrm{s}$ and permeability $k=7.4 \times 10^{-11} \mathrm{~m}^{2}$ estimated from the Carman-Kozeny relation. The impact of the quadratic in time $t^{2}$ term which depends on the molecular diffusion and permeability is to reduce the dispersion relative to the linear behavior. Reprinted figure with permission from T.R. Brosten, S.J. Vogt, J.D. Seymour, S.L. Codd, R.S. Maier, Phys. Rev. E 85, 045301(R) (2012). Copyright 2012 by the American Physical Society.

established approach for preasymptotic Taylor dispersion in a capillary $[17,54]$ and dispersion in turbulent flow [55]. PGSE NMR has been demonstrated to characterize the time dependent Taylor dispersion in capillary flow in excellent agreement with the theory $[38,54,56]$. The reader is directed to Brosten et al. and Saffman for details of the treatment $[53,55]$. In summary, the expansion in time based on that of Saffman results in a short-time quadratic $t^{2}$ scaling of dispersion coefficient with $\left\langle\nabla^{2} v\right\rangle^{2}$, i.e., $D(t) \propto$ $\left\langle\nabla^{2} v\right\rangle^{2} t^{2}[55]$. In low $R e$ Stokes flow $\mu \nabla^{2} v=\nabla p$ resulting in the scaling $\left\langle\nabla^{2} v\right\rangle^{2} \propto\left\langle v_{\| \mid}\right\rangle^{2} / k$ from Darcy's law [53]. The result of the approach applied to low Re macroscopically homogeneous porous media is the trace of the shorttime preasymptotic dispersion coefficient which is given by $D(t)=D_{\mathrm{o}}(t)+\left\langle u^{2}\right\rangle t / 6-\left\langle v_{\|}\right\rangle^{2} \varepsilon \kappa t^{2} / 18 k+\ldots$ [53]. Here $D_{\mathrm{o}}(t)$ is the time dependent effective restricted diffusion due to the molecular diffusion $\kappa=D_{\mathrm{m}}$ sampling of the pore space [57]. Figure 7 shows the difference between the time dependent dispersion and diffusion coefficients normalized by the molecular diffusion $\left[D(t)-D_{\mathrm{o}}(t)\right] / \kappa$ as measured by PGSE NMR for $P e=50$ compared to the linear and quadratic time fits of the preasymptotic dispersion coefficient [53]. The behavior of the dispersion as plotted in Figure 7 shows elegant dependence on $P e$ with the contribution of the permeability term varying with $P e$ as shown in Brosten et al. [53]. The data demonstrate the short-time decrease in dispersion dynamics due to the negative sign on the quadratic term which is dependent on the molecular diffusion and the permeability. This decrease in short-time dispersion due to the interaction of molecular diffusion and the permeability of the porous media is analogous to the impact of molecular diffusion on hydrodynamic dispersion in turbulent flow [55]. The linear behavior shown by the dashed line corresponds to the simple ballistic motion of the OUT process as at short times. The PGSE NMR data is in exact agreement with the reduced dispersion coefficient predicted by the negative sign of the $t^{2}$ quadratic in time term dependent on the permeability.

\section{Conclusions}

In this paper the measurement of preasymptotic hydrodynamic dispersion using displacement time dependent PGSE NMR techniques has been overviewed. The displacement scale dependent hydrodynamic dispersion dynamics obtained from the low-order moments of the displacement probability distribution, or propagator, provide a means to characterize the transport length scale in porous media and permeability. Analysis of the second moment of the displacement dynamics in the long displacement, low- $q$, regime allows isolation of the time dependence of the dispersion from the displacement length, or wavelength $q$, dependence simplifying interpretation. The ability to characterize a transport-based structural length scale from the preasymptotic transverse dispersion behavior is demonstrated in consolidated granular sphere packings and solid open cell foam porous media, systems with drastically differing porosity, permeability and topological complexity. Further application of the methods discussed in this overview, to more geometrically and topologically complex natural porous media exhibiting fractal and multiscale heterogeneity, is an open research topic. The connection between porous media models based on Brownian motion theory, random capillary networks and generalized Langevin memory function equations of nonequilibrium statistical mechanics and the PGSE NMR data provides an experimental basis for testing the applicability of these theories. The PGSE NMR data open up new avenues for development of nonequilibrium statistical mechanics memory function models which incorporate specific details of pore structure. This has potential to enhance the design of porous media to control transport processes in a range of applications through further understanding of models which relate structure and transport.

The authors acknowledge funding from NSF CAREER Award CBET-0642328 (SLC) and DOE OS BER DE-FG02-07-ER64416 (JDS and SLC). The authors thank Dr. R.S. Maier for many helpful discussions on porous media transport. The authors acknowledge Dr. T.R. Brosten for collaboration 
and for making Figure 5. The authors acknowledge the late Prof. Sir P.T. Callaghan for guidance and support.

\section{References}

1. Y.-Q. Song, New J. Physics 13 (2011)

2. J.-P. Korb, New J. Physics 13, 035016 (2011)

3. L.F. Gladden, J. Mitchell, New J. Physics 13, 035001 (2011)

4. Y.-Q. Song, H. Cho, T. Hopper, A.E. Pomerantz, P.Z. Sun, J. Chem. Phys. 128, 052212 (2008)

5. P.T. Callaghan, Translational Dynamics and Magnetic Resonance Principles of Pulsed Gradient Spin Echo NMR (Oxford University Press, New York, 2011)

6. S.P. Neuman, D.M. Tartakovsky, Adv. Water Resour. 32 , $670(2009)$

7. A.E. Scheidegger, J. Appl. Phys. 25, 994 (1954)

8. P.G. Saffman, J. Fluid Mech. 6, 321 (1959)

9. J.H. Cushman, B.X. Hu, T.R. Ginn, J. Stat. Phys. 75, 859 (1994)

10. J. Koplik, S. Redner, D. Wilkinson, Phys. Rev. A 37, 2619 (1988)

11. M. Sahimi, Flow and Transport in Porous Media and Fractured Rock: From Classical methods to Modern Approaches (VCH, Weinheim, 1995)

12. B. Berkowitz, A. Cortis, M. Dentz, H. Scher, Rev. Geophys. 44, RG2003 (2006)

13. H.M. Jaeger, S.R. Nagel, R.P. Behringer, Rev. Mod. Phys. 68, 1259 (1996)

14. R. Kubo, M. Toda, N. Hashitsume, Statistical Physics II: Nonequilibrium Statistical Mechanics (Springer-Verlag, Berlin, 1991)

15. G.I. Taylor, Proc. Lond. Math. Soc. 20, 196 (1921)

16. G.I. Taylor, Proc. R. Soc. B, Series A: Math. Phys. Sci. 219, 186 (1953)

17. C. van den Broeck, Physica A: Stat. Theor. Phys. (Amsterdam) 168, 677 (1990)

18. H. Brenner, J. Stat. Phys. 62, 1095 (1991)

19. P.G. Saffman, J. Fluid Mech. 7, 194 (1960)

20. R. Zwanzig, Nonequilibrium Statistical Mechanics (Oxford University Press, New York, 2001)

21. D.L. Koch, J.F. Brady, Phys. Fluids 31, 965 (1988)

22. J. Salles, J.F. Thovert, R. Delannay, L. Prevors, J.L. Auriault, P.M. Adler, Phys. Fluids A Fluid Dyn. 5, 2348 (1993)

23. J.D. Seymour, P.T. Callaghan, AIChE J. 43, 2096 (1997)

24. B. Bijeljic, M.J. Blunt, Water Resour. Res. 42, W01202 (2006)

25. B. Bijeljic, M.J. Blunt, Water Resour. Res. 43, W12S11 (2007)

26. J.-P. Boon, S. Yip, Molecular Hydrodynamics (McGraw-Hill, New York, 1980)

27. W. Ebeling, I.M. Sokolov, Statistical Thermodynamics and Stochastic Theory of Nonequilibrium Systems (World Scientific Publishing Co., London, 2005)
28. B.J. Berne, J.P. Boon, S.A. Rice, J. Chem. Phys. 45, 1086 (1966)

29. G.K. Batchelor, An Introduction to Fluid Dynamics (Cambridge University Press, Cambridge, 1967)

30. P.T. Callaghan, S.L. Codd, Phys. Fluids 13, 412 (2001)

31. T.R. Brosten, Ph.D. Dissertation, Montana State University, 2009

32. T.R. Brosten, S.L. Codd, R.S. Maier, J.D. Seymour, Phys. Rev. Lett. 103, 218001 (2009)

33. S. Stapf, K.J. Packer, R.G. Graham, J.-F. Thovert, P.M. Adler, Phys. Rev. E 58, 6206 (1998)

34. M.W. Hunter, P.T. Callaghan, Phys. Rev. Lett. 99, 210602 (2007)

35. G. Zheng, W.S. Price, J. Magn. Reson. 195, 40 (2008)

36. Y.E. Kutsovsky, L.E. Scriven, H.T. Davis, B.E. Hammer, Phys. Fluids 8, 863 (1996)

37. L. Lebon, J. Leblond, J.P. Hulin, Phys. Fluids 9, 481 (1997)

38. P.T. Callaghan, S.L. Codd, J.D. Seymour, Concepts Magn. Reson. 11, 181 (1999)

39. J.D. Seymour, P.T. Callaghan, J. Magn. Reson. Series A 122, 90 (1996)

40. R.S. Maier, D.M. Kroll, Y.E. Kutsovsky, H.T. Davis, R.S. Bernard, Phys. Fluids 10, 60 (1998)

41. G.P. Wadsworth, J.G. Bryan, Introduction to Probabiliity and Random Variables (McGraw-Hill, New York, 1960)

42. U.M. Scheven, P.N. Sen, Phys. Rev. Lett. 89, 254501 (2002)

43. J. Stepisnik, Prog. NMR Spectrosc. 17, 187 (1985)

44. B. Manz, P. Alexander, L.F. Gladden, Phys. Fluids 11, $259(1999)$

45. R.S. Maier, D.M. Kroll, R.S. Bernard, S.E. Howington, J.F. Peters, H.T. Davis, Phys. Fluids 12, 2065 (2000)

46. U.M. Scheven, R. Harris, M.L. Johns, Phys. Rev. Lett. 99, $054502(2007)$

47. R.S. Maier, D.M. Kroll, R.S. Bernard, S.E. Howington, J.F. Peters, H.T. Davis, Phys. Fluids 15, 3795 (2003)

48. R.S. Maier, D.M. Kroll, H.T. Davis, AIChE J. 53, 527 (2007)

49. P.T. Callaghan, J. Stepisnik, Adv. Magn. Opt. Reson. 19 325 (1996)

50. T.R. Brosten, S.L. Codd, R.S. Maier, J.D. Seymour, Phys. Fluids 23, 093105 (2011)

51. M.D. Montminy, A.R. Tannenbaum, C.W. Macosko, J. Colloid Interface Sci. 280, 202 (2004)

52. D.L. Johnson, J. Koplik, L.M. Schwartz, Phys. Rev. Lett. 57, $2564(1986)$

53. T.R. Brosten, S.J. Vogt, J.D. Seymour, S.L. Codd, R.S. Maier, Phys. Rev. E 85, 045301(R) (2012)

54. C. van den Broeck, Physica 112A, 343 (1982)

55. P.G. Saffman, J. Fluid Mech. 8, 273 (1960)

56. S.L. Codd, B. Manz, J.D. Seymour, P.T. Callaghan, Phys. Rev. E 60, R3491 (1999)

57. P.N. Sen, Concepts Magn. Reson. Part A 23A, 1 (2004) 